\title{
Model of Spare Parts Optimization Based on System Supply Availability
}

\author{
Linjie Duan 1, a, Bo Xu 1,b, Jinggang Cao ${ }^{1, c}$, Xuewen Wang ${ }^{2, d}$ \\ ${ }^{1}$ Petrochina Pipeline R\&D Center, Langfang, China \\ 2 Petrochina Pipeline shenyang Oil Transportation Sub-company, Shenyang, China \\ aduanlj01@petrochina.com.cn, bkjxubo@petrochina.com.cn, ccaojg01@petrochina.com.cn, \\ d1113710062@qq.com
}

\begin{abstract}
Keywords: Spare Parts; Optimization Model; Supply Availability; Marginal Effect
Abstract. Spare parts optimization which means finding the number of spare parts to achieve the target reliability value provided that the minimum cost and other system reliability conditions are satisfied is of crucial importance in industry. In the paper, the mathematic model for spare parts optimization is established based on system availability and the constraints are set as the practical engineering requirements. The marginal effect analysis method is then applied in optimization model. Further the improved Barlow optimization algorithm is used to obtain the optimal configuration of spare parts. Application results show that an optimized initial configuration scheme is obtained and all the constraints are satisfied including minimum cost and achieving the target system reliability value.
\end{abstract}

\section{Introduction}

The spare parts are crucial support for the system to perform tasks and prevent incidents. Besides, it is also one of the important factors affecting the system reliability. At present, the spare parts as important material foundation for system maintenance. However, it always faces dual contradiction in actual security tasks. On one hand, if the number of spare parts is too small, it will not be able to satisfy the system maintenance on time in case of emergency, which could cause the loss of system integrity, and even affect the execution of the tasks. On the other hand, too many backlog of spare parts will undoubtedly result in the waste of resources and storage space.

The essential purpose of spare parts optimization is to search the optimal configuration to make the system task reliability highest and meet the minimum requirement of reliability under the condition of limited cost.

There are several methods for calculating spare parts amount [1 4], most of which calculate the amount of each spare parts based on equipment unit reliability under the condition of given probability. However, these methods only consider the calculation of digital number of spare parts at unit-level rather than system-level. Meanwhile, system spare supply availability and the influence of the cost of spare parts are not taken into consideration.

There are many disadvantage of the model of spare parts optimization. To cure the above problems, this paper presents an optimization model of spare parts based on system supply availability, using the marginal effect analysis method to determine the search direction, the various spare parts and the optimal allocation scheme for determining system.

\section{Model of Spare Parts Optimization Based on System Reliability}

The optimization problem of spare parts was firstly proposed by Barlow ${ }^{[1]}$ who made comprehensive study. Then Barlow models and methods were widely applied in related research at domestic domestically and internationally. Barlow model regards the system unit and its spare parts as a cold standby system and the whole spare parts system as a system in the composition of each subsystem series, as shown in Figure 1. 


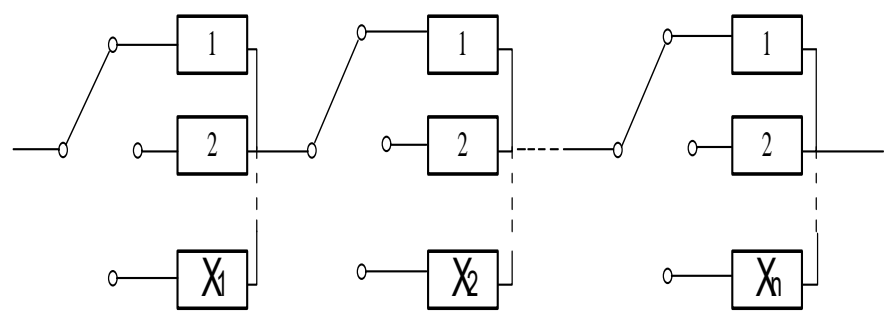

Fig.1 cold-standby system

Barlow took the reserves - the reliability of series system as the security of spare parts. The spare parts optimization of system was defined as: to make the system spare parts purchase cost minimum under the condition of a given system reliability constraints. The specific model is shown as the following:

$$
\left\{\begin{array}{l}
\min \sum_{j=1}^{n} c_{j} x_{j} \\
\prod_{j-1}^{n} R_{j}\left(x_{j}, t_{j}\right) \geq R_{0}
\end{array}\right.
$$

Where $n$ is the type of system unit; $x_{j}$ is the number of the $\mathrm{j}$ kind of spare part; $c_{j}$ is the unit price of the $\mathrm{j}$ kind of spare part; $R_{j}\left(x_{j}, t_{j}\right)$ is spare supporting rate while the $\mathrm{j}$ kind of components equip the $x_{j}$ kind of spare part. $R_{0}$ is the spare supporting rate which is required by the total system?

Barlow model is used as a reliability design model taking the system into consideration which is a straight forward and efficient optimization model in theory and practice. However, while being applied in spare parts optimization in pipeline system, it meets with some difficulties. Due to variety of spare parts in pipeline systems, if this sort of series model is applied to evaluate spare supporting rate of pipeline systems, even if spare supporting rate of each spare part is 0.99 and the number of types is $n=50$, spare supporting rate of the system is only 0.605 , because of the characteristic of the semi system. Apparently, the calculation results cannot match the reality well. In practical maintenance process, there are few spare parts whose spare supporting rate can reach 0.99 or above, needless to say, the total supporting rate of the whole complex system spare parts is so high.

In conclusion, rationality of the evaluation function of spare parts optimization model should be further thought. In fact, there's no reason that pipeline system is considered as a series model, because in practice, when one or a few units do not work and need to be replaced, the appropriate spare parts in storage warehouses can make sure that system can operate well. While, without right spare parts in storage warehouses, it will result in missing parts. In usual, spare supporting rate should be evaluated by supply level of spare parts. As a result, with optimizing spare parts, it ought to be spare parts supply availability rather than system reliability that is regarded as spare supporting rate.

\section{The Improved Model Of Spare Parts Optimization}

In inventory theory, supply availability is:

$$
A_{S}=\frac{M T B M}{M T B M+M S D}
$$

Where MTBM refers to the mean time between maintenance; MSD is the average delay time of spare parts supply

Then system supporting rate is:

$$
R_{j}=\sum_{j=1}^{x_{j}} \frac{\left(N_{j}\right)^{X_{j}}}{x_{j} !} A_{s}
$$

Where $N_{j}$ is the demand of the $\mathrm{j}$ kind of spare parts. 
The purpose of optimizing spare parts is to reduce the amount of spare parts shortage and make the whole cost minimum, under the condition of system spare parts supply availability achieving the maximum.

In the Barlow optimization algorithm, it adopts the method of gradually construct optimal solution, make the optimal decisions which look optimal under certain standards in each stage. Once decision is made, it does not change any more. The rule to make a decision is that: a spare part is added to increase the system reliability each time, but the each unit which is increased is the spare parts whose value is able to provide maximum reliability growth of parts. Search direction function of the system is shown as the following:

$$
\mu_{j}\left(n_{j}\right)=\frac{1}{\text { price }_{j}}\left[\log R_{j}\left(k_{j}+1, t\right)-\log R_{j}\left(k_{j}, t\right)\right]
$$

Where $R_{j}$ is the system reliability; According to the search direction function, the maximum $\mu_{z}\left(n_{z}\right)$ is calculated, and then the next step is to add a part which is the $\mathrm{z}$ kind, and repeat the work until meet the constraint conditions.

Based on the optimization algorithm of Barlow, optimization direction can be constructed under the condition that the availability or cost is the objective function. Through the optimization of the exponential distribution parts, the optimization model of the objective function for maximum security of the system, cost and the security of spare parts for the constraint condition is established as the following:

$$
\begin{gathered}
\operatorname{Max}_{S}=\sum_{j=1}^{n} \frac{\lambda_{j}}{\sum_{j=1}^{n} \lambda_{j}} R_{j} \\
\text { s.t. }\left\{\begin{array}{l}
\sum_{j=1}^{n} c_{j} X_{j} \leq C_{S} \\
R_{1} \leq R_{j} \leq R_{2}
\end{array}\right.
\end{gathered}
$$

Where $R_{j}$ is the system supply availability, as shown in equation (3). Demand of exponential distribution:

$$
N=\lambda t
$$

The idea of marginal effect analysis technique is to increase one spare part in the algorithm every single time in order to find the spare part which can have the biggest cost effectiveness all. If the benefit of adding this spare part is the largest, the number of the spare parts will increase by one. As a result, according to the marginal effect, search direction function of the system is:

$$
\mu_{j}\left(x_{j}\right)=\frac{1}{c_{j}}\left[\frac{\lambda_{j}}{\sum_{i=1}^{n} \lambda_{j}} R_{j}\left(k_{j}+1, t\right)-\frac{\lambda_{j}}{\sum_{j=1}^{n} \lambda_{j}} R_{j}\left(k_{j}, t\right)\right]
$$

The next step is to calculate the $\mu$ value of each component. Because each spare part obeys index distribution here, $\lambda_{i}$ of each spare part is constant. The $\sum_{i=1}^{n} \lambda_{i}$ is a constant, so it does not affect the

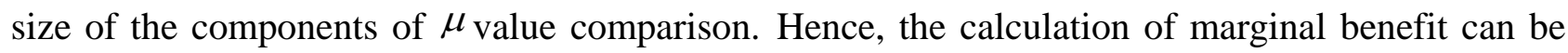
calculated by the following equation:

$$
\mu_{j}\left(x_{j}\right)=\frac{\lambda_{j}}{c_{j}}\left[R_{j}\left(k_{j}+1, t\right)-R_{j}\left(k_{j}, t\right)\right]
$$

Exponential distribution probability density function is shown in figure 2 . 


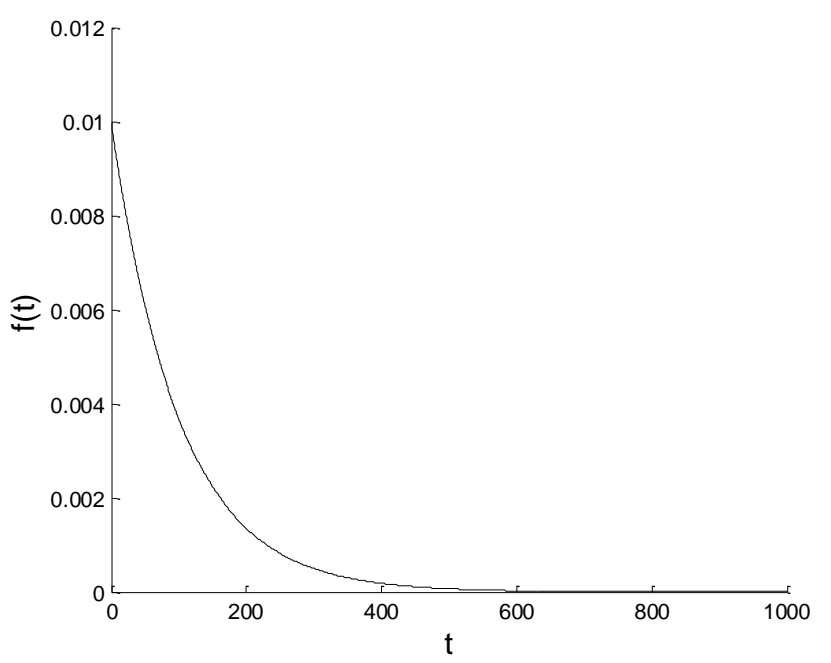

Fig.2 exponential distribution probability density function

In addition, to ensure that each time can diminish marginal effect, the function increase or decrease and bump properties of supporting rate of spare parts need to be analyzed. $R_{j}$ qualification and change with the relationship between the stock, $x_{j}$ curve is shown in figure 3 .

After determining the marginal effect of calculation method and the amount of initial spare parts spare parts, spare parts can be optimized according to the following steps:

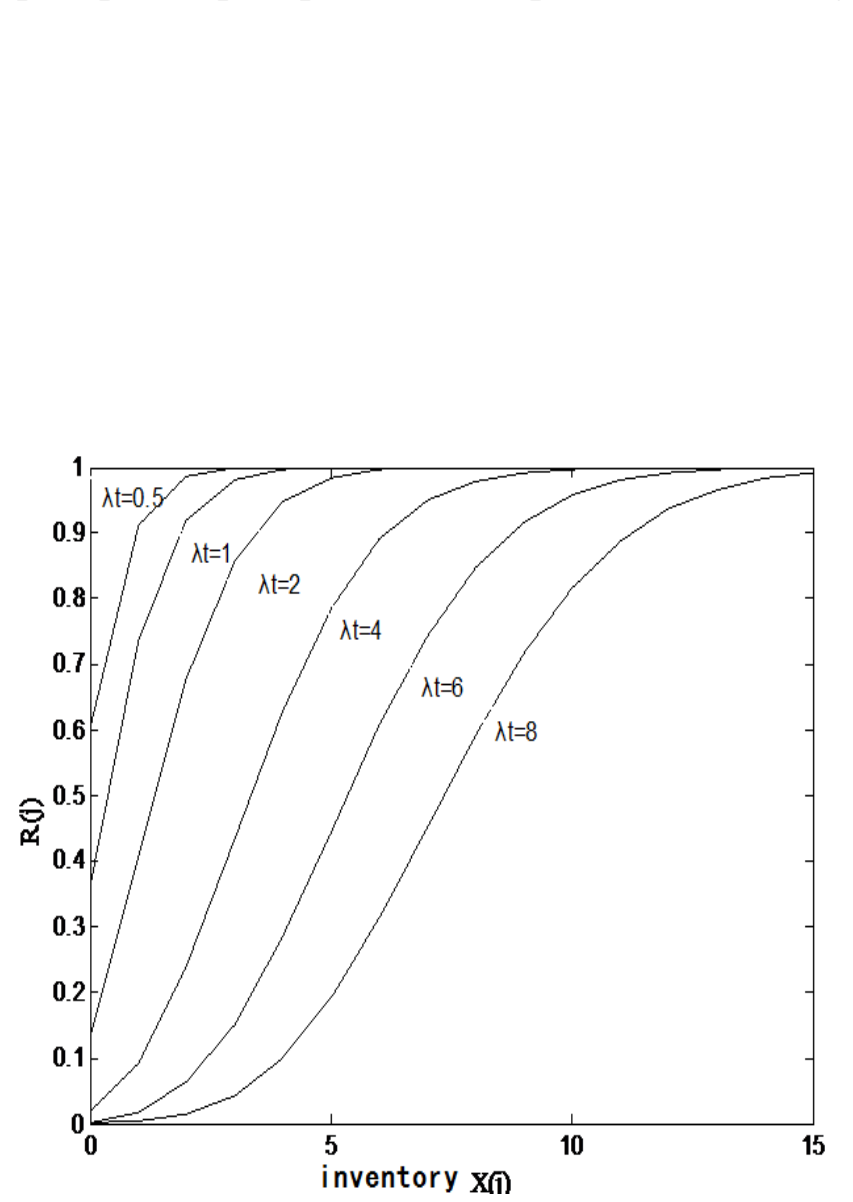

Fig3 along with the security of inventory change curve

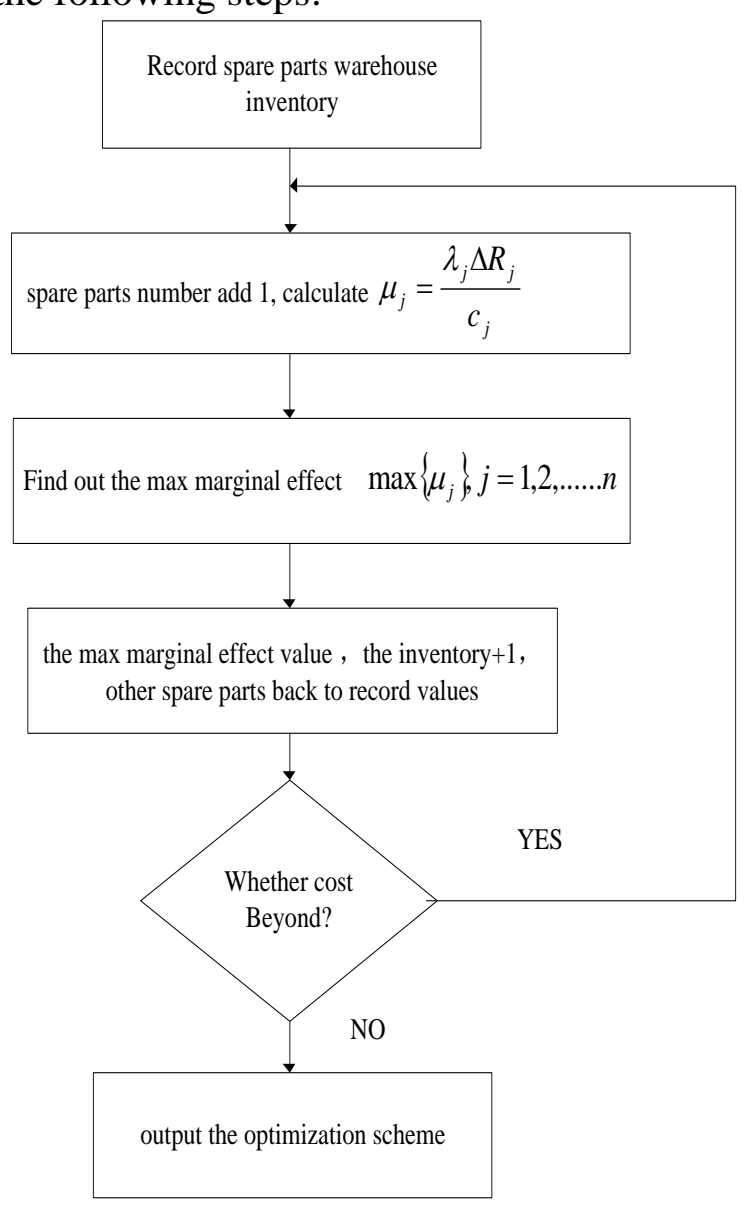

Fig.4 optimization flow chart

\section{Application}

To solve the above mathematical model, the initial condition need to be set and boundary condition equations need to be add to close the system: 
A system consists of six LRU parts, all of components are equally important and both obey exponential distribution. The failure rate and other parameters are shown in Table 1.

Table 1 the LRU parameter values

\begin{tabular}{|c|c|c|}
\hline Order & $\lambda_{j} / h^{-1}$ & Price $c_{j} / \mathrm{RMB}$ \\
\hline LRU1 & $\lambda_{1}=2 \times 10^{-3}$ & 25000 \\
\hline LRU2 & $\lambda_{2}=5.5 \times 10^{-3}$ & 10000 \\
\hline LRU3 & $\lambda_{3}=5 \times 10^{-3}$ & 30000 \\
\hline LRU4 & $\lambda_{4}=2.5 \times 10^{-3}$ & 15000 \\
\hline LRU5 & $\lambda_{5}=3 \times 10^{-3}$ & 13000 \\
\hline LRU6 & $\lambda_{6}=3.5 \times 10^{-3}$ & 5000 \\
\hline
\end{tabular}

Calculation of the demand $\lambda_{j} t$ of turnaround time is shown in Table 2.

Table 2 the turnaround time demand

\begin{tabular}{|c|c|c|c|c|c|c|}
\hline order & LRU1 & LRU2 & LRU3 & LRU4 & LRU5 & LRU6 \\
\hline$\lambda_{j} t$ & 1 & 2.75 & 2.5 & 1.25 & 1.5 & 1.75 \\
\hline
\end{tabular}

Obviously, the demand of LRU2 LRU3 is bigger than 2. It cannot use marginal benefit optimization directly but need to find the convex interval starting point.

For an example of LRU2, which calculates its guarantee rate $R\left(x_{2}\right)$ and marginal benefit $\lambda_{2} \Delta R\left(x_{2}\right) / c_{2}$, is shown in Table 3.

Table 3 supporting rate change table

\begin{tabular}{|c|c|c|c|c|c|c|c|}
\hline$x_{2}$ & 0 & 1 & 2 & 3 & 4 & 5 & 6 \\
\hline$R\left(x_{2}\right)$ & 0.064 & 0.2397 & 0.482 & 0.703 & 0.855 & 0.939 & 0.978 \\
\hline$\frac{\lambda_{2} \Delta R\left(x_{2}\right)}{c_{2}} / 10^{-3}$ & & 0.967 & 1.3299 & 1.218 & 0.838 & 0.461 & 0.211 \\
\hline
\end{tabular}

It is clear that the worth changing trend about marginal benefit of LRU2 is increasing - decreasing. So, the function of $R\left(x_{2}\right)$ is after the first concave convex. And starting with $x_{2}$ value of 2 , the marginal benefit of LRU2 is diminishing. Therefore the initial inventory level is 2 . LRU3 is analyzed by the same method, and initial inventory level of LRU3 is 2 .so before optimizing, initial value of six parts is: $(0,2,2,0,0,0)$.

After determining the initial inventory adjustment quantity of all components, marginal benefit analysis method can be used for optimization of inventory forecast. Marginal effect analysis process is shown in Table 4 (the value of the marginal benefit $\mu$ is $10^{-2}$ ). With the budget of $350000 \mathrm{RMB}$, the model for spare parts inventory systems is:

$$
\left(x_{1}, x_{2}, x_{3}, x_{4}, x_{5}, x_{6}\right)=(1,6,5,3,3,5)
$$

After system being optimized, the value of the security of individual spare parts and spare parts cost are shown in Table 5, the calculated for security of system is 0.945 . The total cost is 344000 $\mathrm{RMB}$; the total number of spare parts is 23 . 
Table 4 the marginal benefit analysis process

\begin{tabular}{|c|c|c|c|c|c|c|c|c|c|c|c|c|}
\hline $\mathrm{xj}^{2}$ & \multicolumn{2}{|c|}{ LRU1 } & \multicolumn{2}{|c|}{ LRU2 } & \multicolumn{2}{|c|}{ LRU3 } & \multicolumn{2}{|c|}{ LRU4 } & \multicolumn{2}{|c|}{ LRU5 } & \multicolumn{2}{|c|}{ LRU6 } \\
\hline 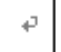 & $R\left(x_{1}\right)$ & $\mu_{1}$, & $R\left(x_{2}\right)$ & $\mu_{2}$ & $R\left(x_{3}\right)$ & $\mu_{3}$ & $R\left(x_{4}\right)$ & $\mu_{4}$ & $R\left(x_{5}\right)$ & $\mu_{5}$ & $R\left(x_{6}\right)$ & $\mu_{6}$ \\
\hline 0 & 0.3679 & 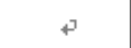 & 0.0639 & P & 0.0821 & 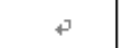 & 0.2865 & 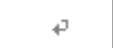 & 0.2231 & 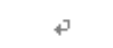 & 0.1738 & 5 \\
\hline 1 & 0.7358 & 0.29432 & 0.2397 & 0.9669 & 0.2873 & 0.342 & 0.6446 & 0.5968 & 0.5578 & 0.7724 & 0.4779 & 2.1287 \\
\hline 2 & 0.9197 & 0.14712 & 0.4815 & 1.3299 & 0.5438 & 0.7125 & 0.8685 & 0.3732 & 0.8088 & 0.5792 & 0.744 & 1.8627 \\
\hline 3 & 0.981 & 0.04904 & 0.703 & 1.21825 & 0.7576 & 0.5939 & 0.9617 & 0.1553 & 0.9344 & 0.2898 & 0.8992 & 1.0864 \\
\hline 4 & 0.9963 & 0.01224 & 0.8554 & 0.8382 & 0.8912 & 0.3711 & ت 0.9909 & 0.0487 & 0.9814 & 0.1085 & 0.9671 & 0.4753 \\
\hline 5 & 0.9994 & 0.00248 & 0.9392 & 0.4609 & 0.958 & 0.1856 & 0.9982 & 0.0122 & 0.9955 & 0.0325 & 0.9909 & 0.1666 \\
\hline 6 & 0.9999 & 0.0004 & 0.9776 & 0.2112 & 0.9858 & 0.0772 & ت 0.9997 & 0.0025 & 0.9991 & 0.0083 & 0.9978 & 0.0483 \\
\hline 7 & 1 & $8 \mathrm{E}-05$ & 0.9927 & 0.08305 & 0.9958 & 0.0278 & 1 & 0.0005 & 0.9998 & 0.0016 & 0.9995 & 0.0119 \\
\hline 8 & 1 & 0 & 0.9978 & 0.02805 & 0.9989 & 0.0086 & 1 & 0 & 1 & 0.0005 & 0.9999 & 0.0028 \\
\hline 9 & 1 & 0 & 0.9994 & 0.0088 & 0.9997 & 0.0022 & 1 & 0 & 1 & 0 & 1 & 0.0007 \\
\hline 10 & 1 & 0 & 0.9999 & 0.00275 & 0.9999 & 0.0006 & 1 & 0 & 1 & 0 & 1 & 0 \\
\hline
\end{tabular}

Table 5 supporting rate and spare parts cost optimization results

\begin{tabular}{|c|c|c|c|c|}
\hline Parts & number $x_{j}$ & price $c_{j} / R M B$ & $R_{j}$ & $\operatorname{cost} C_{j} / R M B$ \\
\hline LRU1 & 1 & 25000 & 0.7358 & 25000 \\
\hline LRU 2 & 6 & 10000 & 0.9776 & 60000 \\
\hline LRU 3 & 5 & 30000 & 0.958 & 150000 \\
\hline LRU 4 & 3 & 15000 & 0.9617 & 45000 \\
\hline LRU 5 & 3 & 13000 & 0.9344 & 39000 \\
\hline LRU 6 & 5 & 5000 & 0.9909 & 25000 \\
\hline
\end{tabular}

It can be found that when the number of spare parts LRU1 is 1, the value of the supporting rate of the spare parts is 0.7358 , less than 0.8. It cannot meet the requirement of the supporting rate of single piece, so it needs to adjust the number of spare parts for LRU1. According to the supporting rate of single spare parts LRU1, minimum number of spare parts should be 2. At this point, after the adjustment makes the security of the system increase to 0.96 , the total cost is $369000 \mathrm{RMB}$; more than $19000 \mathrm{RMB}$ cost constraints. In the case of supporting rate is preferred, the final output of spare parts plan is:

$$
\left(x_{1}, x_{2}, x_{3}, x_{4}, x_{5}, x_{6}\right)=(2,6,5,3,3,5)
$$

In order to compare the optimization effect, in the same conditions, the supporting rate of calculation model for single spare part and the optimized total cost comparison is shown in figure 6 . When a piece of supporting rate of spare parts is 0.945 , the number of parts is:

$$
\left(x_{1}, x_{2}, x_{3}, x_{4}, x_{5}, x_{6}\right)=(3,6,5,3,4,4)
$$

Now the cost is $402000 \mathrm{RMB}$, the total number of spare parts is 25 ; the optimized cost is 369000 $\mathrm{RMB}$, and the total number of spare parts is 24 , cost optimization effect is obvious. 


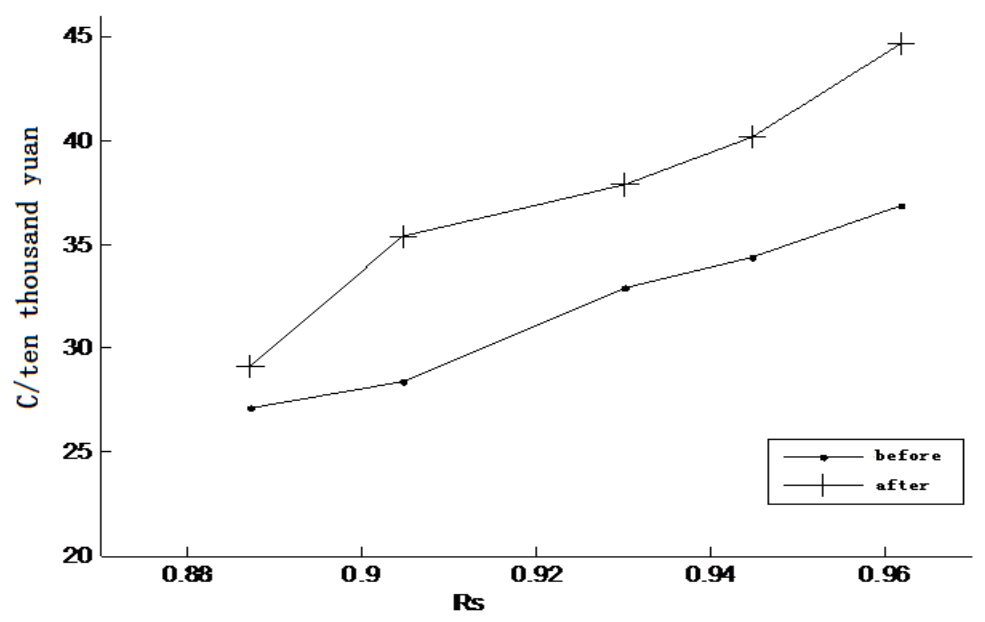

Fig.5 cost comparison of the same supporting rate

\section{Acknowledgements}

In this article, according to the study of Barlow optimization model and optimization algorithm, we found that although the method has the certain superiority in the spare parts optimization, but the system reliability and maintenance of Barlow model is not sure of the equivalent actually. Optimization model is established which based on Barlow optimization algorithm. In this model, it treats spare parts supply system availability as supporting rate. This conforms to the actual production of maintenance. Based on the marginal benefit, search direction and optimization process is given, which is suitable for the optimization model. Finally, an example is given, the analysis results show the effectiveness of the model.

\section{References}

[1] Will main, T.R., Smart, C.N., Schwarz, H.F.A new approach to forecasting intermittent demand for service parts inventories [J]. International Journal of Forecasting, 2004, 20: 375-387..

[2] Gupta. A. Approximate solution of a single-base mufti-in-denture repairable-item inventory system [J]. Journal of the Operational Research Society, 1993, 44: 701-710.

[3] Barlow R Mathematical theory of reliability [M].New York: John Wiley \& sons Inc., 1965.

[4] Srinivasan, V.S.First Emptiness in the Spare Parts Problem for Reparable Components [J]. Operations Research, 1968, 16(2):407-415.

[5] Morris, A.C., Paul.R.K, Hau, L.L. and David F P. Mufti-item service constrained (s, S) policy for spare parts logistics system [J]. Naval Research Logistics. 1992, 39:561-77.

[6] Marcello Bra glia, A.G and Roberto. Multi-attribute classification method for spare parts inventory management [J]. Journal of Quality in Maintenance Engineering. 2004, 10(1):55-65.

[7] Kamath, K., Rajashree Pakkala, T. P. M. A Bayesian approach to a dynamic inventory model under an unknown demand distribution [J]. Computers \& Operations Research, 2002, 29: 403-422.

[8] Gupta. A. Approximate solution of a single-base mufti-in-denture repairable-item inventory system [J]. Journal of the Operational Research Society, 1993, 44: 701-710.

[9] Will main, T.R., Smart, C.N., Schwarz, H.F.A new approach to forecasting intermittent demand for service parts inventories [J]. International Journal of Forecasting, 2004, 20: 375-387. 